Bull. Korean Math. Soc. 51 (2014), No. 5, pp. 1375-1397

http://dx.doi.org/10.4134/BKMS.2014.51.5.1375

\title{
CODIMENSION REDUCTION FOR SUBMANIFOLDS OF UNIT $(4 m+3)$-SPHERE AND ITS APPLICATIONS
}

\author{
Hyang SoOK Kim AND Jin Suk PAK
}

\begin{abstract}
In this paper we establish codimension reduction theorem for submanifolds of a $(4 m+3)$-dimensional unit sphere $S^{4 m+3}$ with Sasakian 3 -structure and apply it to submanifolds of a quaternionic projective space.
\end{abstract}

\section{Introduction}

As is well-known, for a submanifold $M$ of a Riemannian manifold $\widetilde{M}$, the codimension of $M$ is said to be reduced if there exists a totally geodesic submanifold $\bar{M}$ of $\widetilde{M}$ such that $M \subset \bar{M}$.

In particular, when the ambient manifold is a complex manifold, the intermediate submanifold $\bar{M}$ is requested to be not only totally geodesic, but also complex submanifold.

The codimension reduction problem was investigated by Allendoerfer [1] in the case that the ambient manifold $\widetilde{M}$ is a Euclidean space and by Erbacher [14] in the case that $\widetilde{M}$ is a real space form. For submanifolds of a complex projective space, Cecil [2] proved a codimension reduction theorem for complex submanifolds. Okumura [14] extended Cecil's result to real submanifolds by using the standard submersion method established by Lawson [12] (for real submanifolds of a complex hyperbolic space, see [8]).

As a quaternionic analogue for real submanifolds of a quaternionic projective space, Kwon and the second author [11] provided a codimension reduction theorem which may correspond to Okumura's result in [14] (for real submanifolds of a quaternionic hyperbolic space, see [9]).

On the other hand, in 1982, Okumura [13] studied submanifolds $M$ of an odd-dimensional sphere with the canonical Sasakian structure $\{\phi, \xi\}$ to which the structure vector field $\xi$ is always tangent and proved that, under some

Received March 7, 2013.

2010 Mathematics Subject Classification. 53C40, 53C25.

Key words and phrases. codimension reduction, unit $(4 m+3)$-sphere, Sasakian 3structure, normal connection, quaternionic projective space, $L$-flat, mean curvature vector, totally geodesic.

This work was supported by the 2012 Inje University research grant. 
additional conditions, if $\operatorname{dim}\left(T_{x} M \cap \phi T_{x} M^{\perp}\right)$ is less than that codimension, then there exists such a totally geodesic $\phi$-invariant submanifold $\bar{M}$ that $M \subset \bar{M}$, where $T_{x} M$ and $T_{x} M^{\perp}$ denote the tangent space and the normal space to $M$ at $x \in M$, respectively. Using this theorem, in his paper [13], Okumura presented a codimension reduction theorem for real submanifolds of a complex projective space by means of the standard submersion method due to Lawson [12].

In this paper we first consider a $(4 m+3)$-dimensional unit sphere with the canonical Sasakian 3-structure $\{\phi, \psi, \theta\}$ (for definition, see $[7,10,17]$ ). Let $M$ be a real submanifold of the space to which the structure vector fields $\xi, \eta, \zeta$ are always tangent. If at each point $x \in M$ the tangent space $T_{x} M$ satisfies

$$
\phi T_{x} M \subset T_{x} M, \quad \psi T_{x} M \subset T_{x} M, \quad \theta T_{x} M \subset T_{x} M,
$$

$M$ is called an invariant submanifold under $\{\phi, \psi, \theta\}$. It is well known that an invariant submanifold is a manifold with Sasakian 3-structure. We consider the more general case that at each point $x \in M T_{x} M$ and $T_{x} M^{\perp}$ satisfy the condition that $\operatorname{dim}\left(T_{x} M \cap \phi T_{x} M^{\perp} \cap \psi T_{x} M^{\perp} \cap \theta T_{x} M^{\perp}\right)$ is independent of $x$. Such submanifolds involve invariant submanifolds as a special case.

The main purpose of the paper is to study relations between $\operatorname{dim}\left(T_{x} M \cap\right.$ $\left.\phi T_{x} M^{\perp} \cap \psi T_{x} M^{\perp} \cap \theta T_{x} M^{\perp}\right)$ and the codimension of $M$, and to prove that, under some additional conditions, if $\operatorname{dim}\left(T_{x} M \cap \phi T_{x} M^{\perp} \cap \psi T_{x} M^{\perp} \cap \theta T_{x} M^{\perp}\right)$ is less than the codimension, then there exists a totally geodesic invariant submanifold $M^{\prime}$ such that $M \subset M^{\prime}$, which will be used in codimension reducing for submanifolds of a quaternionic projective space by using the standard submersion method established by Lawson [12].

All manifolds, submanifolds and geometric objects will be assumed to be connected, differentiable and of class $C^{\infty}$, and all maps also be of class $C^{\infty}$ if not stated otherwise.

\section{Submanifolds of a $(4 m+3)$-dimensional unit sphere}

Let us consider a $(4 m+3)$-dimensional unit sphere $S^{4 m+3}$ as a real hypersurface of the real $4(m+1)$-dimensional quaternionic number space $Q^{m+1}$. For any point $x$ in $S^{4 m+3}$, we set

$$
\xi=E_{1} x, \quad \eta=E_{2} x, \quad \zeta=E_{3} x
$$

where $\left\{E_{1}, E_{2}, E_{3}\right\}$ denotes the canonical quaternionic Kähler structure of $Q^{m+1}$. Then $\{\xi, \eta, \zeta\}$ becomes a Sasakian 3-structure, namely, $\xi, \eta$ and $\zeta$ are mutually orthogonal unit Killing vector fields which satisfy

$$
\begin{gathered}
\bar{\nabla}_{Y} \bar{\nabla}_{X} \xi=g(X, \xi) Y-g(Y, X) \xi, \\
\bar{\nabla}_{Y} \bar{\nabla}_{X} \eta=g(X, \eta) Y-g(Y, X) \eta, \\
\bar{\nabla}_{Y} \bar{\nabla}_{X} \zeta=g(X, \zeta) Y-g(Y, X) \zeta
\end{gathered}
$$

for any vector fields $X, Y$ tangent to $S^{4 m+3}$, where $g$ denotes the canonical metric on $S^{4 m+3}$ induced from that of $Q^{m+1}$ and $\bar{\nabla}$ the Riemannian connection 
with respect to $g$. In this case, putting

$$
\phi X=\bar{\nabla}_{X} \xi, \quad \psi X=\bar{\nabla}_{X} \eta, \quad \theta X=\bar{\nabla}_{X} \zeta
$$

it follows that

$$
\begin{gathered}
\phi \xi=0, \psi \eta=0, \theta \zeta=0, \\
\psi \zeta=-\theta \eta=\xi, \theta \xi=-\phi \zeta=\eta, \phi \eta=-\psi \xi=\zeta, \\
{[\eta, \zeta]=-2 \xi,[\zeta, \xi]=-2 \eta,[\xi, \eta]=-2 \zeta,} \\
\phi^{2}=-I+f_{\xi} \otimes \xi, \quad \psi^{2}=-I+f_{\eta} \otimes \eta, \quad \theta^{2}=-I+f_{\zeta} \otimes \zeta, \\
\psi \theta=\phi+f_{\zeta} \otimes \eta, \quad \theta \phi=\psi+f_{\xi} \otimes \zeta, \quad \phi \psi=\theta+f_{\eta} \otimes \xi, \\
\theta \psi=-\phi+f_{\eta} \otimes \zeta, \quad \phi \theta=-\psi+f_{\zeta} \otimes \xi, \quad \psi \phi=-\theta+f_{\xi} \otimes \eta,
\end{gathered}
$$

where $I$ denotes the identity transformation and

$$
f_{\xi}(X)=g(\xi, X), \quad f_{\eta}(X)=g(\eta, X), \quad f_{\zeta}(X)=g(\zeta, X) .
$$

Moreover, from (2.1) and (2.2), we have

$$
\begin{aligned}
& \left(\bar{\nabla}_{Y} \phi\right) X=g(X, \xi) Y-g(Y, X) \xi, \quad\left(\bar{\nabla}_{Y} \psi\right) X=g(X, \eta) Y-g(Y, X) \eta, \\
& \left(\bar{\nabla}_{Y} \theta\right) X=g(X, \zeta) Y-g(Y, X) \zeta
\end{aligned}
$$

for any vector fields $X, Y$ tangent to $S^{4 m+3}$ (cf. [7, 10, 15, 17]).

Let $M$ be an $(n+3)$-dimensional submanifold isometrically immersed in $S^{4 m+3}$ and denote by $T M$ and $T M^{\perp}$ the tangent and normal bundle of $M$, respectively. We shall delete the isometric immersion $\tilde{\iota}: M \rightarrow S^{4 m+3}$ and its differential $\iota_{*}$ in our notations. Let $\nabla$ and $\nabla^{\perp}$ denote the covariant differentiation in $M$ and the normal connection of $M$ in $S^{4 m+3}$, respectively. To each $N_{x} \in T_{x} M^{\perp}$, we extend $N_{x}$ to a normal vector field $N$ defined in a neighborhood of $x$. Given an orthonormal basis $\left\{\left(N_{1}\right)_{x}, \ldots,\left(N_{p}\right)_{x}\right\}$ of $T_{x} M^{\perp}$, we denote by $H_{A}$ the Weingarten map with respect to $N_{A}$, which will be called the second fundamental tensor associated to $N_{A}$. If the second fundamental tensors $H_{A}(A=1, \ldots, p)$ vanish identically on $M, M$ is called a totally geodesic submanifold. The first normal space $N_{x}^{1}$ is defined to be the orthogonal complement of $\left\{N_{x} \in T_{x} M^{\perp} \mid H_{N}=0\right\}$ in $T_{x} M^{\perp}$ (cf. [4]). If $N_{1}, \ldots, N_{p}$ are orthonormal normal vector fields in a neighborhood of $x \in M$, they determine normal connection forms $s_{A B}$ in a neighborhood of $x$ by

$$
\nabla_{X}^{\perp} N_{A}=\sum_{B=1}^{p} s_{A B}(X) N_{B}
$$

for $X$ tangent to $M$. Then we have the following Gauss and Weingarten formulas:

$$
\bar{\nabla}_{X} Y=\nabla_{X} Y+\sum_{A=1}^{p} g\left(H_{A} X, Y\right) N_{A}, \quad g\left(H_{A} X, Y\right)=g\left(X, H_{A} Y\right),
$$




$$
\bar{\nabla}_{X} N_{A}=-H_{A} X+\sum_{B=1}^{p} s_{A B}(X) N_{B}, \quad s_{A B}(X)=-s_{B A}(X) .
$$

The mean curvature vector field $\mu$ of $M$ is defined by

$$
\mu=\frac{1}{n+3} \sum_{A=1}^{p}\left(\operatorname{trace} H_{A}\right) N_{A} .
$$

The submanifold $M$ is said to be minimal if $\mu$ vanishes identically on $M$. Differentiating (2.9) covariantly, we have

$$
(n+3) \nabla_{X}^{\perp} \mu=\sum_{A=1}^{p}\left\{\left(X \operatorname{trace} H_{A}\right) N_{A}+\sum_{B=1}^{p}\left(\operatorname{trace} H_{A}\right) s_{A B}(X) N_{B}\right\} .
$$

Hence the mean curvature vector field is parallel with respect to the normal connection $\nabla^{\perp}$ if and only if

$$
X\left(\operatorname{trace} H_{A}\right)=\sum_{B=1}^{p}\left(\operatorname{trace} H_{B}\right) s_{A B}(X) .
$$

Let us denote by $R$ and $R^{N}$ the curvature tensors for $\nabla$ and $\nabla^{\perp}$, respectively. Since the curvature tensor $\bar{R}$ for $\bar{\nabla}$ on $S^{4 m+3}$ is given by

$$
\bar{R}(X, Y) Z=g(Y, Z) X-g(X, Z) Y,
$$

we have the following relations:

$$
\begin{aligned}
& \left(\nabla_{X} H_{A}\right) Y-\left(\nabla_{Y} H_{A}\right) X=\sum_{B=1}^{p}\left\{s_{A B}(X) H_{B} Y-s_{A B}(Y) H_{B} X\right\}, \\
& R^{N}(X, Y) N_{A}=\sum_{B=1}^{p} g\left(\left(H_{A} H_{B}-H_{B} H_{A}\right) X, Y\right) .
\end{aligned}
$$

If $R^{N}$ vanishes identically on $M$, the normal connection of $M$ in $S^{4 m+3}$ is said to be flat. The normal connection of $M$ is flat if and only if $H_{A} H_{B}=H_{B} H_{A}$ for all $A, B=1,2, \ldots, p$ (cf. [3]).

For any $X \in T M$ and for $N_{A}, A+1,2, \ldots, p$, the transforms $\phi X, \psi X, \theta X$ and $\phi N_{A}, \psi N_{A}, \theta N_{A}$ are, respectively, written in the following forms:
(i) $\phi X=F X+\sum_{A=1}^{p} u^{A}(X) N_{A}$,
(ii) $\psi X=G X+\sum_{A=1}^{p} v^{A}(X) N_{A}$,
(iii) $\theta X=H X+\sum_{A=1}^{p} w^{A}(X) N_{A}$,
(i) $\phi N_{A}=-U_{A}+\sum_{B=1}^{p} P_{A B}^{\phi} N_{B}$,
(ii) $\psi N_{A}=-V_{A}+\sum_{B=1}^{p} P_{A B}^{\psi} N_{B}$, 
(iii) $\theta N_{A}=-W_{A}+\sum_{A=1}^{p} P_{A B}^{\theta} N_{B}$,

where $\{F, G, H\}$ and $\left\{P^{\phi}, P^{\psi}, P^{\theta}\right\}$ define endomorphisms of $T M$ and $T M^{\perp}$, respectively, and $\left\{U_{A}, V_{A}, W_{A}\right\}$ and $\left\{u^{A}, v^{A}, w^{A}\right\}$ are local tangent vector fields and local 1-forms on $M$. They satisfy

$$
\begin{aligned}
& g(F X, Y)=-g(X, F Y), \quad g(G X, Y)=-g(X, G Y), \\
& g(H X, Y)=-g(X, H Y), \\
& P_{A B}^{\phi}=-P_{B A}^{\phi}, \quad P_{A B}^{\psi}=-P_{B A}^{\psi}, \quad P_{A B}^{\theta}=-P_{B A}^{\theta}, \\
& u^{A}(X)=g\left(U_{A}, X\right), \quad v^{A}(X)=g\left(V_{A}, X\right), \quad w^{A}(X)=g\left(W_{A}, X\right)
\end{aligned}
$$

for tangent vectors $X, Y$ to $M$. If $U_{A}=0, V_{A}=0, W_{A}=0, A=1,2, \ldots, p$ identically, the submanifold is called an invariant submanifold under $\{\phi, \psi, \theta\}$.

In what follows we assume that the Sasakian 3-structure vector fields $\xi, \eta, \zeta$ are always tangent to $M$ and use the same notations as appeared in the case of ambient manifold. Then, from (2.3), (2.4) and (2.13), we have

(2.18) $F \xi=0, \quad G \eta=0, \quad H \zeta=0$,

(2.19) $F \eta=\zeta, \quad F \zeta=-\eta, \quad G \zeta=\xi, \quad G \xi=-\zeta, \quad H \xi=\eta, \quad H \eta=-\xi$,

(2.20) $u^{A}(\xi)=u^{A}(\eta)=u^{A}(\zeta)=0, \quad v^{A}(\xi)=v^{A}(\eta)=v^{A}(\zeta)=0$,

$$
w^{A}(\xi)=w^{A}(\eta)=w^{A}(\zeta)=0, \quad A=1,2, \ldots, p .
$$

Applying $\phi$ to both sides of $(2.13)_{(\mathrm{i})}$ and $(2.14)_{(\mathrm{i})}$, it follows from (2.4), (2.5), (2.13)-(2.14) and (2.16)-(2.17) that

$$
\begin{aligned}
& F^{2} X=-X+\sum_{A=1}^{p} u^{A}(X) U_{A}+g(\xi, X) \xi, \quad F U_{A}=-\sum_{B=1}^{p} P_{A B}^{\phi} U_{B}, \\
& g\left(U_{A}, U_{B}\right)=\delta_{A B}+\sum_{C=1}^{p} P_{A C}^{\phi} P_{C B}^{\phi}
\end{aligned}
$$

because the structure vector field $\xi$ is tangent to $M$. Similarly, from $(2.13)_{\text {(ii) }}$, $(2.13)_{(\mathrm{iii})},(2.14)_{(\mathrm{ii})}$ and $(2.14)_{(\mathrm{iii})}$, we get

$$
\begin{aligned}
& G^{2} X=-X+\sum_{A=1}^{p} v^{A}(X) V_{A}+g(\eta, X) \eta, \quad G V_{A}=-\sum_{B=1}^{p} P_{A B}^{\psi} V_{B} \\
& g\left(V_{A}, V_{B}\right)=\delta_{A B}+\sum_{C=1}^{p} P_{A C}^{\psi} P_{C B}^{\psi} \\
& H^{2} X=-X+\sum_{A=1}^{p} w^{A}(X) W_{A}+g(\zeta, X) \zeta, \quad H W_{A}=-\sum_{B=1}^{p} P_{A B}^{\theta} W_{B} \\
& g\left(W_{A}, W_{B}\right)=\delta_{A B}+\sum_{C=1}^{p} P_{A C}^{\theta} P_{C B}^{\theta} .
\end{aligned}
$$


Applying $\psi$ and $\theta$ to both sides of $(2.13)_{(\mathrm{i})}$, respectively, and using (2.3)-(2.5), (2.13)-(2.14) and (2.16)-(2.17), we have

$$
\begin{aligned}
& G F X=-H X+\sum_{A=1}^{p} u^{A}(X) V_{A}+g(\xi, X) \eta, \\
& v^{A}(F X)=-w^{A}(X)+\sum_{B=1}^{p} P_{A B}^{\psi} u^{B}(X), \\
& H F X=G X+\sum_{A=1}^{p} u^{A}(X) W_{A}+g(\xi, X) \zeta, \\
& w^{A}(F X)=v^{A}(X)+\sum_{B=1}^{p} P_{A B}^{\theta} u^{B}(X) .
\end{aligned}
$$

Similarly, it follows from $(2.13)_{(\mathrm{ii})}$ and $(2.13)_{(\mathrm{iii})}$ that

$$
\begin{aligned}
& H G X=-F X+\sum_{A=1}^{p} v^{A}(X) W_{A}+g(\eta, X) \zeta, p \\
& w^{A}(G X)=-u^{A}(X)+\sum_{B=1}^{p} P_{A B}^{\theta} v^{B}(X), \\
& F G X=H X+\sum_{A=1}^{p} v^{A}(X) U_{A}+g(\eta, X) \xi \\
& u^{A}(G X)=w^{A}(X)+\sum_{B=1}^{p} P_{A B}^{\phi} v^{B}(X), \\
& F H X=-G X+\sum_{A=1}^{p} w^{A}(X) U_{A}+g(\zeta, X) \xi \\
& u^{A}(H X)=-v^{A}(X)+\sum_{B=1}^{p} P_{A B}^{\phi} w^{B}(X), \\
& G H X=F X+\sum_{A=1}^{p} w^{A}(X) V_{A}+g(\zeta, X) \eta \\
& v^{A}(H X)=u^{A}(X)+\sum_{B=1}^{p} P_{A B}^{\psi} w^{B}(X) .
\end{aligned}
$$

Applying $\psi$ and $\theta$ to both sides of (2.14) $(\mathrm{i})$, respectively, and using (2.4)(2.5), (2.13)-(2.14) and (2.17), we have

$$
G U_{A}=-W_{A}-\sum_{B=1}^{p} P_{A B}^{\phi} V_{B}, \quad g\left(U_{A}, V_{B}\right)=P_{A B}^{\theta}+\sum_{C=1}^{p} P_{A C}^{\phi} P_{C B}^{\psi},
$$


(2.31) $H U_{A}=V_{A}-\sum_{B=1}^{p} P_{A B}^{\phi} W_{B}, \quad g\left(U_{A}, W_{B}\right)=-P_{A B}^{\psi}+\sum_{C=1}^{p} P_{A C}^{\phi} P_{C B}^{\theta}$.

Similarly, it follows from $(2.14)_{(i i)}$ and $(2.14)_{(i i i)}$ that

$$
\begin{aligned}
& H V_{A}=-U_{A}-\sum_{B=1}^{p} P_{A B}^{\psi} W_{B}, \quad g\left(V_{A}, W_{B}\right)=P_{A B}^{\phi}+\sum_{C=1}^{p} P_{A C}^{\psi} P_{C B}^{\theta} \\
& F V_{A}=W_{A}-\sum_{B=1}^{p} P_{A B}^{\psi} U_{B}, \quad g\left(V_{A}, U_{B}\right)=-P_{A B}^{\theta}+\sum_{C=1}^{p} P_{A C}^{\psi} P_{C B}^{\phi} \\
& F W_{A}=-V_{A}-\sum_{B=1}^{p} P_{A B}^{\theta} U_{B}, \quad g\left(W_{A}, U_{B}\right)=P_{A B}^{\psi}+\sum_{C=1}^{p} P_{A C}^{\theta} P_{C B}^{\phi} \\
& G W_{A}=U_{A}-\sum_{B=1}^{p} P_{A B}^{\theta} V_{B}, \quad g\left(W_{A}, V_{B}\right)=-P_{A B}^{\phi}+\sum_{C=1}^{p} P_{A C}^{\theta} P_{C B}^{\psi}
\end{aligned}
$$

Differentiating $(2.13)_{(\mathrm{i})}$ covariantly and making use of (2.6)-(2.8), (2.13)(2.14) and (2.16), we obtain

$$
\begin{aligned}
\left(\nabla_{Y} F\right) X= & g(X, \xi) Y-g(X, Y) \xi-\sum_{A=1}^{p} g\left(H_{A} X, Y\right) U_{A} \\
& +\sum_{A=1}^{p} u^{A}(X) H_{A} Y, \\
\left(\nabla_{Y} u^{A}\right) X= & -g\left(H_{A} F X, Y\right)-\sum_{B=1}^{p} P_{A B}^{\phi} g\left(H_{B} X, Y\right) \\
& +\sum_{B=1}^{p} s_{A B}(Y) u^{B}(X) .
\end{aligned}
$$

Similarly, from $(2.13)_{(\mathrm{ii})}$ and $(2.13)_{(\mathrm{iii})}$, we also get

$$
\begin{aligned}
\left(\nabla_{Y} G\right) X= & g(X, \eta) Y-g(X, Y) \eta-\sum_{A=1}^{p} g\left(H_{A} X, Y\right) V_{A} \\
& +\sum_{A=1}^{p} v^{A}(X) H_{A} Y \\
\left(\nabla_{Y} v^{A}\right) X= & -g\left(H_{A} G X, Y\right)-\sum_{B=1}^{p} P_{A B}^{\psi} g\left(H_{B} X, Y\right) \\
& +\sum_{B=1}^{p} s_{A B}(Y) v^{B}(X),
\end{aligned}
$$




$$
\begin{aligned}
\left(\nabla_{Y} H\right) X= & g(X, \zeta) Y-g(X, Y) \zeta-\sum_{A=1}^{p} g\left(H_{A} X, Y\right) W_{A} \\
& +\sum_{A=1}^{p} w^{A}(X) H_{A} Y, \\
\left(\nabla_{Y} w^{A}\right) X= & -g\left(H_{A} H X, Y\right)-\sum_{B=1}^{p} P_{A B}^{\theta} g\left(H_{B} X, Y\right) \\
& +\sum_{B=1}^{p} s_{A B}(Y) w^{B}(X) .
\end{aligned}
$$

Differentiating $(2.14)_{(\mathrm{i})}$ covariantly and taking account of (2.6)-(2.8), (2.13)(2.14) and (2.16), we obtain

$$
\begin{aligned}
\nabla_{X} U_{A}= & F H_{A} X-\sum_{B=1}^{p} P_{A B}^{\phi} H_{B} X+\sum_{B=1}^{p} s_{A B}(X) U_{B}, \\
\nabla_{X} P_{A B}^{\phi}= & g\left(U_{A}, H_{B} X\right)-u^{B}\left(H_{A} X\right)-\sum_{C=1}^{p} P_{A C}^{\phi} s_{C B}(X) \\
& +\sum_{C=1}^{p} P_{B C}^{\phi} s_{C A}(X) .
\end{aligned}
$$

Similarly, from $(2.13)_{(i i)}$ and $(2.13)_{(i i i)}$, we also get

$$
\begin{aligned}
\nabla_{X} V_{A}= & G H_{A} X-\sum_{B=1}^{p} P_{A B}^{\psi} H_{B} X+\sum_{B=1}^{p} s_{A B}(X) V_{B}, \\
\nabla_{X} P_{A B}^{\psi}= & g\left(V_{A}, H_{B} X\right)-v^{B}\left(H_{A} X\right)-\sum_{C=1}^{p} P_{A C}^{\psi} s_{C B}(X) \\
& +\sum_{C=1}^{p} P_{B C}^{\psi} s_{C A}(X), \\
\nabla_{X} W_{A}= & H H_{A} X-\sum_{B=1}^{p} P_{A B}^{\theta} H_{B} X+\sum_{B=1}^{p} s_{A B}(X) W_{B}, \\
\nabla_{X} P_{A B}^{\theta}= & g\left(W_{A}, H_{B} X\right)-w^{B}\left(H_{A} X\right)-\sum_{C=1}^{p} P_{A C}^{\theta} s_{C B}(X) \\
& +\sum_{C=1}^{p} P_{B C}^{\theta} s_{C A}(X) .
\end{aligned}
$$

Moreover, it is clear from (2.2) that

$$
\nabla_{X} \xi=F X, \quad \nabla_{X} \eta=G X, \quad \nabla_{X} \zeta=H X,
$$




$$
H_{A} \xi=U_{A}, \quad H_{A} \eta=V_{A}, \quad H_{A} \zeta=W_{A} .
$$

\section{Laplacian for a global function defined on $M$}

We define a function $f$ on $M$ by

$$
f=\sum_{A=1}^{p}\left\{u^{A}\left(U_{A}\right)+v^{A}\left(V_{A}\right)+w^{A}\left(W_{A}\right)\right\} .
$$

Then, since $\xi, \eta, \zeta$ are mutually orthogonal unit vector fields, (2.21)-(2.23) yield

$$
f=\operatorname{tr} F^{2}+\operatorname{tr} G^{2}+\operatorname{tr} H^{2}+3(n-1), \quad(\operatorname{tr}:=\operatorname{trace})
$$

which means that $f$ is independent of the choice of $N_{A}{ }^{\prime} s$ and thus $f$ is a global function defined on $M . \quad f$ vanishes identically on $M$ if and only if $M$ is an invariant submanifold under $\{\phi, \psi, \theta\}$.

From now on we compute the Laplacian $\Delta f$. For any vector field $X$ on $M$ it follows from (2.15), (2.17)-(2.18), (2.36), (2.38) and (2.40) that

$$
\begin{aligned}
\frac{1}{2} X f & =\frac{1}{2} X\left(\operatorname{tr} F^{2}+\operatorname{tr} G^{2}+\operatorname{tr} H^{2}\right) \\
& =\operatorname{tr}\left(\nabla_{X} F\right) F+\operatorname{tr}\left(\nabla_{X} G\right) G+\operatorname{tr}\left(\nabla_{X} H\right) H \\
& =2 \sum_{A=1}^{p}\left\{g\left(F H_{A} X, U_{A}\right)+g\left(G H_{A} X, V_{A}\right)+g\left(H H_{A} X, W_{A}\right)\right\},
\end{aligned}
$$

from which together with (2.20)-(2.23), (2.42), (2.44), (2.46) and (2.49), we get

$$
\begin{aligned}
& \frac{1}{4}\left(\nabla_{Y} \nabla_{X} f-\nabla_{\nabla_{Y} X} f\right)=\frac{1}{4}\left\{\nabla_{Y}(X f)-\left(\nabla_{Y} X\right) f\right\} \\
= & \sum_{A=1}^{p}\left\{g\left(\left(\nabla_{Y} F\right) H_{A} X, U_{A}\right)+g\left(F\left(\nabla_{Y} H_{A}\right) X, U_{A}\right)+g\left(F H_{A} X, \nabla_{Y} U_{A}\right)\right. \\
& +g\left(\left(\nabla_{Y} G\right) H_{A} X, V_{A}\right)+g\left(G\left(\nabla_{Y} H_{A}\right) X, V_{A}\right)+g\left(G H_{A} X, \nabla_{Y} V_{A}\right) \\
& +g\left(\left(\nabla_{Y} H\right) H_{A} X, W_{A}\right)+g\left(H\left(\nabla_{Y} H_{A}\right) X, W_{A}\right)+g\left(H H_{A} X, \nabla_{Y} W_{A}\right) \\
= & \sum_{A=1}^{p}\left[g\left(U_{A}, X\right) g\left(U_{A}, Y\right)+g\left(V_{A}, X\right) g\left(V_{A}, Y\right)+g\left(W_{A}, X\right) g\left(W_{A}, Y\right)\right. \\
& -g\left(\left(\nabla_{Y} H_{A}\right) F U_{A}, X\right)-g\left(\left(\nabla_{Y} H_{A}\right) G V_{A}, X\right)-g\left(\left(\nabla_{Y} H_{A}\right) H W_{A}, X\right) \\
& -g\left(H_{A} F^{2} H_{A} X, Y\right)-g\left(H_{A} G^{2} H_{A} X, Y\right)-g\left(H_{A} H^{2} H_{A} X, Y\right) \\
& +\sum_{B=1}^{p}\left\{g\left(H_{A} U_{B}, X\right) g\left(H_{B} U_{A}, Y\right)+g\left(H_{A} V_{B}, X\right) g\left(H_{B} V_{A}, Y\right)\right. \\
& +g\left(H_{A} W_{B}, X\right) g\left(H_{B} W_{A}, Y\right)-g\left(H_{B} H_{A} X, Y\right) g\left(U_{B}, U_{A}\right) \\
& -g\left(H_{B} H_{A} X, Y\right) g\left(V_{B}, V_{A}\right)-g\left(H_{B} H_{A} X, Y\right) g\left(W_{B}, W_{A}\right) \\
& -P_{A B}^{\phi} g\left(H_{B} F H_{A} X, Y\right)-P_{A B}^{\psi} g\left(H_{B} G H_{A} X, Y\right)
\end{aligned}
$$




$$
\begin{aligned}
& -P_{A B}^{\theta} g\left(H_{B} H H_{A} X, Y\right)+s_{A B}(Y) g\left(F H_{A} X, U_{B}\right) \\
& \left.\left.+s_{A B}(Y) g\left(G H_{A} X, V_{B}\right)+s_{A B}(Y) g\left(H H_{A} X, W_{B}\right)\right\}\right] .
\end{aligned}
$$

On the other hand, substituting $F U_{A}, G V_{A}$ and $H W_{A}$ for $X$ into (2.11), respectively, we have

$$
\begin{gathered}
\left(\nabla_{Y} H_{A}\right) F U_{A}=\left(\nabla_{F U_{A}} H_{A}\right) Y+\sum_{B=1}^{p}\left\{s_{A B}(Y) H_{B} F U_{A}-s_{A B}\left(F U_{A}\right) H_{B} Y\right\}, \\
\left(\nabla_{Y} H_{A}\right) G V_{A}=\left(\nabla_{G V_{A}} H_{A}\right) Y+\sum_{B=1}^{p}\left\{s_{A B}(Y) H_{B} G V_{A}-s_{A B}\left(G V_{A}\right) H_{B} Y\right\}, \\
\left(\nabla_{Y} H_{A}\right) H W_{A}=\left(\nabla_{H W_{A}} H_{A}\right) Y+\sum_{B=1}^{p}\left\{s_{A B}(Y) H_{B} H W_{A}-s_{A B}\left(H W_{A}\right) H_{B} Y\right\},
\end{gathered}
$$

which together with (3.2) yield

$$
\begin{aligned}
& \frac{1}{4}\left(\nabla_{Y} \nabla_{X} f-\nabla_{\nabla_{Y}} f\right) \\
=\sum_{A=1}^{p}[g & \left(U_{A}, X\right) g\left(U_{A}, Y\right)+g\left(V_{A}, X\right) g\left(V_{A}, Y\right)+g\left(W_{A}, X\right) g\left(W_{A}, Y\right) \\
& -g\left(\left(\nabla_{F U_{A}} H_{A}\right) Y, X\right)-g\left(\left(\nabla_{G V_{A}} H_{A}\right) Y, X\right)-g\left(\left(\nabla_{H W_{A}} H_{A}\right) Y, X\right) \\
& -g\left(H_{A} F^{2} H_{A} X, Y\right)-g\left(H_{A} G^{2} H_{A} X, Y\right)-g\left(H_{A} H^{2} H_{A} X, Y\right) \\
+\sum_{B=1}^{p} & \left\{s_{A B}\left(F U_{A}\right) g\left(H_{B} Y, X\right)+s_{A B}\left(G V_{A}\right) g\left(H_{B} Y, X\right)\right. \\
& +s_{A B}\left(H W_{A}\right) g\left(H_{B} Y, X\right)+g\left(H_{A} U_{B}, X\right) g\left(H_{B} U_{A}, Y\right) \\
& +g\left(H_{A} V_{B}, X\right) g\left(H_{B} V_{A}, Y\right)+g\left(H_{A} W_{B}, X\right) g\left(H_{B} W_{A}, Y\right) \\
& \quad-g\left(H_{B} H_{A} X, Y\right) g\left(U_{B}, U_{A}\right)-g\left(H_{B} H_{A} X, Y\right) g\left(V_{B}, V_{A}\right) \\
& -g\left(H_{B} H_{A} X, Y\right) g\left(W_{B}, W_{A}\right)-P_{A B}^{\phi} g\left(H_{B} F H_{A} X, Y\right) \\
& \left.\left.-P_{A B}^{\psi} g\left(H_{B} G H_{A} X, Y\right)-P_{A B}^{\theta} g\left(H_{B} H H_{A} X, Y\right)\right\}\right] .
\end{aligned}
$$

Hence we have

$$
\begin{aligned}
& \frac{1}{4} \Delta f=\sum_{A=1}^{p}[ g\left(U_{A}, U_{A}\right)+g\left(V_{A}, V_{A}\right)+g\left(W_{A}, W_{A}\right)-\operatorname{tr} F^{2} H_{A}^{2}-\operatorname{tr} G^{2} H_{A}^{2} \\
&- \operatorname{tr} H^{2} H_{A}^{2}-\nabla_{F U_{A}}\left(\operatorname{tr} H_{A}\right)-\nabla_{G V_{A}}\left(\operatorname{tr} H_{A}\right)-\nabla_{H W_{A}}\left(\operatorname{tr} H_{A}\right) \\
&+\sum_{B=1}^{p}\left\{s_{A B}\left(F U_{A}\right) \operatorname{tr} H_{B}+s_{A B}\left(G V_{A}\right) \operatorname{tr} H_{B}+s_{A B}\left(H W_{A}\right) \operatorname{tr} H_{B}\right. \\
& \quad+g\left(H_{A} U_{B}, H_{B} U_{A}\right)+g\left(H_{A} V_{B}, H_{B} V_{A}\right)+g\left(H_{A} W_{B}, H_{B} W_{A}\right) \\
&-\left(\operatorname{tr} H_{B} H_{A}\right) g\left(U_{B}, U_{A}\right)-\left(\operatorname{tr} H_{B} H_{A}\right) g\left(V_{B}, V_{A}\right)
\end{aligned}
$$




$$
\begin{aligned}
& -\left(\operatorname{tr} H_{B} H_{A}\right) g\left(W_{B}, W_{A}\right)-P_{A B}^{\phi}\left(\operatorname{tr} F H_{A} H_{B}\right) \\
& \left.\left.-P_{A B}^{\psi}\left(\operatorname{tr} G H_{A} H_{B}\right)-P_{A B}^{\theta}\left(\operatorname{tr} H H_{A} H_{B}\right)\right\}\right] .
\end{aligned}
$$

On the other hand, (2.21)-(2.23) and (2.49) imply

$$
\begin{gathered}
\operatorname{tr} F^{2} H_{A}^{2}=-\operatorname{tr} H_{A}^{2}+g\left(U_{A}, U_{A}\right)+\sum_{B=1}^{p} g\left(H_{A} U_{B}, H_{A} U_{B}\right), \\
\operatorname{tr} G^{2} H_{A}^{2}=-\operatorname{tr} H_{A}^{2}+g\left(V_{A}, V_{A}\right)+\sum_{B=1}^{p} g\left(H_{A} V_{B}, H_{A} V_{B}\right), \\
\operatorname{tr} H^{2} H_{A}^{2}=-\operatorname{tr} H_{A}^{2}+g\left(W_{A}, W_{A}\right)+\sum_{B=1}^{p} g\left(H_{A} W_{B}, H_{A} W_{B}\right),
\end{gathered}
$$

from which combined with (3.3) it follows that

$$
\begin{aligned}
\frac{1}{4} \Delta f=\sum_{A=1}^{p}[3 & \operatorname{tr} H_{A}^{2}-\left(F U_{A}\right) \operatorname{tr} H_{A}-\left(G V_{A}\right) \operatorname{tr} H_{A}-\left(H W_{A}\right) \operatorname{tr} H_{A} \\
+\sum_{B=1}^{p} & \left\{s_{A B}\left(F U_{A}\right) \operatorname{tr} H_{B}+s_{A B}\left(G V_{A}\right) \operatorname{tr} H_{B}+s_{A B}\left(H W_{A}\right) \operatorname{tr} H_{B}\right. \\
& +g\left(H_{A} U_{B}, H_{B} U_{A}-H_{A} U_{B}\right)+g\left(H_{A} V_{B}, H_{B} V_{A}-H_{A} V_{B}\right) \\
& +g\left(H_{A} W_{B}, H_{B} W_{A}-H_{A} W_{B}\right)-\left(\operatorname{tr} H_{B} H_{A}\right) g\left(U_{B}, U_{A}\right) \\
& -\left(\operatorname{tr} H_{B} H_{A}\right) g\left(V_{B}, V_{A}\right)-\left(\operatorname{tr} H_{B} H_{A}\right) g\left(W_{B}, W_{A}\right) \\
& \left.\left.-P_{A B}^{\phi}\left(\operatorname{tr} F H_{A} H_{B}\right)-P_{A B}^{\psi}\left(\operatorname{tr} G H_{A} H_{B}\right)-P_{A B}^{\theta}\left(\operatorname{tr} H H_{A} H_{B}\right)\right\}\right] .
\end{aligned}
$$

Now we prepare some lemmas for later use.

Lemma 3.1. Let $M$ be a submanifold of a unit $(4 m+3)$-sphere $S^{4 m+3}$ to which the Sasakian 3-structure vector fields $\xi, \eta, \zeta$ are always tangent. If the normal connection of $M$ in $S^{4 m+3}$ is flat, then

$$
\sum_{A=1}^{p} u^{A}\left(U_{A}\right), \quad \sum_{A=1}^{p} v^{A}\left(V_{A}\right), \quad \sum_{A=1}^{p} w^{A}\left(W_{A}\right)
$$

are constant and consequently the function $f$ is also constant.

Proof. For any vector field $X$ tangent to $M$, it follows from (2.7), (2.15), (2.21) and (2.42) that

$$
\begin{aligned}
\frac{1}{2} X\left(\sum_{A=1}^{p} u^{A}\left(U_{A}\right)\right) & =\sum_{A=1}^{p} g\left(\nabla_{X} U_{A}, U_{A}\right) \\
& =\sum_{A=1}^{p}\left[\left\{g\left(F H_{A} X, U_{A}\right)-\sum_{B=1}^{p} P_{A B}^{\phi} g\left(X, H_{B} U_{A}\right)\right]\right.
\end{aligned}
$$




$$
=\sum_{A, B=1}^{p} P_{A B}^{\phi} g\left(X, H_{A} U_{B}-H_{B} U_{A}\right) .
$$

On the other hand, if the normal connection is flat, then by means of (2.49) we obtain

$$
\begin{aligned}
& H_{A} U_{B}-H_{B} U_{A}=\left(H_{A} H_{B}-H_{B} H_{A}\right) \xi=0, \\
& H_{A} V_{B}-H_{B} V_{A}=\left(H_{A} H_{B}-H_{B} H_{A}\right) \eta=0, \\
& H_{A} W_{B}-H_{B} W_{A}=\left(H_{A} H_{B}-H_{B} H_{A}\right) \zeta=0,
\end{aligned}
$$

which together with the above equation yield $X\left(\sum_{A=1}^{p} u^{A}\left(U_{A}\right)\right)=0$, namely $\sum_{A=1}^{p} u^{A}\left(U_{A}\right)$ is constant. Similarly we can prove that $\sum_{A=1}^{p} v^{A}\left(V_{A}\right)$ and $\sum_{A=1}^{p} w^{A}\left(W_{A}\right)$ are also constant.

Lemma 3.2. Let $M$ be as in Lemma 3.1. If the normal connection of $M$ in $S^{4 m+3}$ is flat and the mean curvature vector field $\mu$ is parallel with respect to the normal connection, then

$$
\begin{gathered}
3 \sum_{A=1}^{p} \operatorname{tr} H_{A}^{2}=\sum_{A, B=1}^{p}\left\{\left(\operatorname{tr} H_{A} H_{B}\right) g\left(U_{A}, U_{B}\right)+\left(\operatorname{tr} H_{A} H_{B}\right) g\left(V_{A}, V_{B}\right)\right. \\
\left.+\left(\operatorname{tr} H_{A} H_{B}\right) g\left(W_{A}, W_{B}\right)\right\} .
\end{gathered}
$$

Proof. Owing to Lemma 3.1, it follows from (2.10), (3.4) and (3.5) that

$$
\begin{aligned}
3 \sum_{A=1}^{p} \operatorname{tr} H_{A}^{2}=\sum_{A, B=1}^{p}\{ & \left(\operatorname{tr} H_{B} H_{A}\right) g\left(U_{B}, U_{A}\right)+\left(\operatorname{tr} H_{B} H_{A}\right) g\left(V_{B}, V_{A}\right) \\
& +\left(\operatorname{tr} H_{B} H_{A}\right) g\left(W_{B}, W_{A}\right)+P_{A B}^{\phi}\left(\operatorname{tr} F H_{A} H_{B}\right) \\
& \left.+P_{A B}^{\psi}\left(\operatorname{tr} G H_{A} H_{B}\right)+P_{A B}^{\theta}\left(\operatorname{tr} H H_{A} H_{B}\right)\right\}
\end{aligned}
$$

from which combined with (2.16) and $H_{A} H_{B}=H_{B} H_{A}$, we get (3.6).

\section{Submanifolds with $\operatorname{dim}\left(T M \cap \phi T M^{\perp} \cap \psi T M^{\perp} \cap \theta T M^{\perp}\right)<p$}

Suppose that at a point $x \in M$

$$
\operatorname{dim}\left(T_{x} M \cap \phi T_{x} M^{\perp} \cap \psi T_{x} M^{\perp} \cap \theta T_{x} M^{\perp}\right)=q .
$$

Then we can choose in $T M^{\perp} 3 q$ orthonormal normal vector fields $N_{\alpha}(\alpha=$ $1, \ldots, 3 q)$ in such a way that

$$
\phi_{x}\left(N_{\alpha}\right)_{x}, \psi_{x}\left(N_{\alpha}\right)_{x}, \theta_{x}\left(N_{\alpha}\right)_{x} \in T_{x} M \oplus \operatorname{Span}\left\{N_{\alpha}\right\}_{\alpha=1, \ldots, 3 q},
$$

and further

(4.1)

$$
\phi_{x}\left(N_{1}\right)_{x}=\psi_{x}\left(N_{q+1}\right)_{x}=\theta_{x}\left(N_{2 q+1}\right)_{x}, \ldots, \phi_{x}\left(N_{q}\right)_{x}=\psi_{x}\left(N_{2 q}\right)_{x}=\theta_{x}\left(N_{3 q}\right)_{x} .
$$


In fact, if $\left\{\left(X_{1}\right)_{x}, \ldots,\left(X_{q}\right)_{x}\right\}$ is an orthonormal basis of $T_{x} M \cap \phi T_{x} M^{\perp} \cap$ $\psi T_{x} M^{\perp} \cap \theta T_{x} M^{\perp}$, then there exist $3 q$ normal vector fields $N_{\alpha}$ such that

$$
\begin{aligned}
& \left(X_{1}\right)_{x}=-\phi_{x}\left(N_{1}\right)_{x}=-\psi_{x}\left(N_{q+1}\right)_{x}=-\theta_{x}\left(N_{2 q+1}\right)_{x}, \ldots, \\
& \left(X_{q}\right)_{x}=-\phi_{x}\left(N_{q}\right)_{x}=-\psi_{x}\left(N_{2 q}\right)_{x}=-\theta_{x}\left(N_{3 q}\right)_{x}
\end{aligned}
$$

and consequently all of $\left(X_{i}\right)_{x}$ are mutually orthogonal to $\xi, \eta$ and $\zeta$ because of (2.3). With such a choice of $N_{\alpha}(\alpha=1, \ldots, 3 q)$, it follows from (2.14) that

$$
\begin{aligned}
& \left(X_{1}\right)_{x}=\left(U_{1}\right)_{x}=\left(V_{q+1}\right)_{x}=\left(W_{2 q+1}\right)_{x}, \\
& \quad \vdots \\
& \left(X_{q}\right)_{x}=\left(U_{q}\right)_{x}=\left(V_{2 q}\right)_{x}=\left(W_{3 q}\right)_{x}, \\
& \left(U_{q+1}\right)_{x}=\cdots=\left(U_{3 q}\right)_{x}=0, \\
& \left(V_{1}\right)_{x}=\cdots=\left(V_{q}\right)_{x}=\left(V_{2 q+1}\right)_{x}=\cdots=\left(V_{3 q}\right)_{x}=0, \\
& \left(W_{1}\right)_{x}=\cdots=\left(W_{2 q}\right)_{x}=0, \\
& P_{(q+1)(2 q+1)}^{\phi}=-P_{(2 q+1)(q+1)}^{\phi}=1, \ldots, P_{(2 q)(3 q)}^{\phi}=-P_{(3 q)(2 q)}^{\phi}=1, \\
& P_{(1)(2 q+1)}^{\psi}=-P_{(2 q+1)(1)}^{\psi}=-1, \ldots, P_{(q)(3 q)}^{\psi}=-P_{(3 q)(q)}^{\psi}=-1, \\
& P_{(1)(q+1)}^{\theta}=-P_{(q+1)(1)}^{\psi}=1, \ldots, P_{(q)(2 q)}^{\theta}=-P_{(2 q)(q)}^{\psi}=1, \\
& P_{\alpha \nu}^{\phi}=0, \quad P_{\alpha \nu}^{\psi}=0, \quad P_{\alpha \nu}^{\theta}=0,(\alpha=1, \ldots, 3 q, \nu=3 q+1, \ldots, p), \\
& \phi_{x}\left(N_{\nu}\right)_{x}=-\left(U_{\nu}\right)_{x}+\sum_{\delta=3 q+1}^{p} P_{\nu \delta}^{\phi}(x)\left(N_{\delta}\right)_{x}, \\
& \psi_{x}\left(N_{\nu}\right)_{x}=-\left(V_{\nu}\right)_{x}+\sum_{\delta=3 q+1}^{p} P_{\nu \delta}^{\psi}(x)\left(N_{\delta}\right)_{x}, \\
& \theta_{x}\left(N_{\nu}\right)_{x}=-\left(W_{\nu}\right)_{x}+\sum_{\delta=3 q+1}^{p} P_{\nu \delta}^{\theta}(x)\left(N_{\delta}\right)_{x},
\end{aligned}
$$

where we have used (2.4) and (4.2). Furthermore, it is clear from (2.4), (4.1) and (4.2) that

$$
\begin{gathered}
g_{x}\left(\left(X_{i}\right)_{x},\left(U_{\nu}\right)_{x}\right)=0, \quad g_{x}\left(\left(X_{i}\right)_{x},\left(V_{\nu}\right)_{x}\right)=0, \quad g_{x}\left(\left(X_{i}\right)_{x},\left(W_{\nu}\right)_{x}\right)=0, \\
i=1, \ldots, q, \quad \nu=3 q+1, \ldots, p .
\end{gathered}
$$

Lemma 4.1. If the normal connection is flat, $q$ is constant over $M$.

Proof. We put

$$
f_{1}=\sum_{A=1}^{p} u^{A}\left(U_{A}\right), \quad f_{2}=\sum_{A=1}^{p} v^{A}\left(V_{A}\right), \quad f_{3}=\sum_{A=1}^{p} w^{A}\left(W_{A}\right) .
$$

Assume that at $y \in M$

$$
\operatorname{dim}\left(T_{y} M \cap \phi T_{y} M^{\perp} \cap \psi T_{y} M^{\perp} \cap \theta T_{y} M^{\perp}\right)=q^{\prime}
$$


and let say $q<q^{\prime}$. At $x$ and $y$ the function $f_{1}$ can be rewritten as the following:

$$
\begin{aligned}
& f_{1}(x)=\sum_{\alpha=1}^{3 q} u^{\alpha}\left(U_{\alpha}\right)(x)+\sum_{\nu=3 q+1}^{3 q^{\prime}} u^{\nu}\left(U_{\nu}\right)(x)+\sum_{\nu=3 q^{\prime}+1}^{p} u^{\nu}\left(U_{\nu}\right)(x), \\
& f_{1}(y)=\sum_{\alpha=1}^{3 q^{\prime}} u^{\alpha}\left(U_{\alpha}\right)(y)+\sum_{\nu=3 q^{\prime}+1}^{p} u^{\nu}\left(U_{\nu}\right)(y) .
\end{aligned}
$$

By means of Lemma 3.1, the function $f_{1}$ is constant and consequently (4.3) and (4.5) imply

$$
3 q+\sum_{\nu=3 q+1}^{3 q^{\prime}} u^{\nu}\left(U_{\nu}\right)(x)+\sum_{\nu=3 q^{\prime}+1}^{p} u^{\nu}\left(U_{\nu}\right)(x)=3 q^{\prime}+\sum_{\nu=3 q^{\prime}+1}^{p} u^{\nu}\left(U_{\nu}\right)(y),
$$

or equivalently,

$$
3\left(q-q^{\prime}\right)+\sum_{\nu=3 q+1}^{3 q^{\prime}} u^{\nu}\left(U_{\nu}\right)(x)+\sum_{\nu=3 q^{\prime}+1}^{p}\left\{u^{\nu}\left(U_{\nu}\right)(x)-u^{\nu}\left(U_{\nu}\right)(y)\right\}=0 .
$$

On the other hand, it follows from (2.21) that $u^{\nu}\left(U_{\nu}\right)=1-\sum_{A=1}^{p}\left(P_{\nu A}^{\phi}\right)^{2}$ and thus

$$
\sum_{\nu=3 q+1}^{3 q^{\prime}} u^{A}\left(U_{A}\right)(x)=3\left(q^{\prime}-q\right)-\sum_{\nu=3 q+1}^{3 q^{\prime}} \sum_{A=1}^{p}\left(P_{\nu A}^{\phi}\right)^{2}(x),
$$

from which, inserting back into (4.6), we have

$$
-\sum_{\nu=3 q+1}^{3 q^{\prime}} \sum_{A=1}^{p}\left(P_{\nu A}^{\phi}\right)^{2}(x)+\sum_{\nu=3 q^{\prime}+1}^{p}\left\{u^{\nu}\left(U_{\nu}\right)(x)-u^{\nu}\left(U_{\nu}\right)(y)\right\}=0 .
$$

Since $u^{\nu}\left(U_{\nu}\right)$ and $P_{\nu A}^{\phi}$ are differentiable functions, we obtain

$$
\lim _{x \rightarrow y}\left\{u^{\nu}\left(U_{\nu}\right)(x)-u^{\nu}\left(U_{\nu}\right)(y)\right\}=0 .
$$

Hence it is clear from (4.7) that

$$
\sum_{A=1}^{p}\left(P_{\nu A}^{\phi}\right)^{2}(y)=0 \text {, i.e., } P_{\nu A}^{\phi}(y)=0, \nu=q+1, \ldots, q^{\prime},
$$

which is a contradiction because of (4.3b). By using the functions $f_{2}$ or $f_{3}$ we can derive the same conclusion.

In the following we assume that $3 q<p$ and that the mean curvature vector field $\mu$ is parallel with respect to the normal connection. Then (2.21)-(2.23), 
(3.6), (4.3) and (4.4) yield

$$
\begin{aligned}
& \sum_{\nu=3 q+1}^{p}\left(\operatorname{tr} H_{\nu}^{2}\right)\left[\left\{1-g\left(U_{\nu}, U_{\nu}\right)\right\}+\left\{1-g\left(V_{\nu}, V_{\nu}\right)\right\}+\left\{1-g\left(W_{\nu}, W_{\nu}\right)\right\}\right] \\
= & \sum_{\nu=3 q+1}^{p}\left(\operatorname{tr} H_{\nu}^{2}\right) \sum_{A=1}^{p}\left\{\left(P_{\nu A}^{\phi}\right)^{2}+\left(P_{\nu A}^{\psi}\right)^{2}+\left(P_{\nu A}^{\theta}\right)^{2}\right\}=0,
\end{aligned}
$$

which implies $\operatorname{tr} H_{\nu}^{2}=0$ for $\nu=3 q+1, \ldots, p$. Thus $H_{\nu}=0, \nu=3 q+1, \ldots, p$ and $U_{\nu}=V_{\nu}=W_{\nu}=0, \nu=3 q+1, \ldots, p$ by means of (2.49). Particularly, when $q=0$, we have the following.

Theorem 4.2. Let $M$ be an $(n+3)$-dimensional complete submanifold isometrically immersed in a unit $(4 m+3)$-sphere $S^{4 m+3}$ to which the structure vector fields $\xi, \eta, \zeta$ are always tangent. Suppose that the normal connection of $M$ in $S^{4 m+3}$ is flat and that the mean curvature vector field is parallel with respect to the normal connection. If $\operatorname{dim}\left(T_{x} M \cap \phi T_{x} M^{\perp} \cap \psi T_{x} M^{\perp} \cap \theta T_{x} M^{\perp}\right)=0$ at some point $x \in M$, then $M$ is a totally geodesic, invariant submanifold and consequently a great sphere.

Corollary 4.3. Let $M$ be an $(n+3)$-dimensional complete, minimal submanifold isometrically immersed in a unit $(4 m+3)$-sphere $S^{4 m+3}$ to which the structure vector fields $\xi, \eta, \zeta$ are always tangent. Suppose that the normal connection of $M$ in $S^{4 m+3}$ is flat and that the mean curvature vector field is parallel with respect to the normal connection. If $\operatorname{dim}\left(T_{x} M \cap \phi T_{x} M^{\perp} \cap \psi T_{x} M^{\perp} \cap \theta T_{x} M^{\perp}\right)=0$ at some point $x \in M$, then $M$ is a totally geodesic, invariant submanifold and consequently a great sphere.

On the other side, in order to consider the case where $0<3 q<p$, we will prepare the following two Lemmas.

Lemma 4.4. For $\alpha=1, \ldots, 3 q$ and $\nu=3 q+1, \ldots, p, s_{\nu \alpha}=0$.

Proof. Since $U_{\nu}=V_{\nu}=W_{\nu}=0$ and $H_{\nu}=0,(2.42),(2.44)$ and (2.46) give

$$
\begin{aligned}
\sum_{B=1}^{p} s_{\nu B} U_{B}= & \sum P_{\nu B}^{\phi} H_{B} X, \quad \sum_{B=1}^{p} s_{\nu B} V_{B}=\sum P_{\nu B}^{\psi} H_{B} X \\
& \sum_{B=1}^{p} s_{\nu B} W_{B}=\sum P_{\nu B}^{\theta} H_{B} X
\end{aligned}
$$

from which together with $P_{\alpha \nu}^{\phi}=P_{\alpha \nu}^{\psi}=P_{\alpha \nu}^{\theta}=0$, it follows that

$$
\sum_{\alpha=1}^{q} s_{\nu \alpha} U_{\alpha}=0, \quad \sum_{\alpha=q+1}^{2 q} s_{\nu \alpha} V_{\alpha}=0, \quad \sum_{\alpha=2 q+1}^{3 q} s_{\nu \alpha} W_{\alpha}=0 .
$$

Hence it is clear from (4.3) that $s_{\nu \alpha}=0$ for $\alpha=1, \ldots, 3 q ; \nu=3 q+1, \ldots, p$. 
Lemma 4.5. The first normal space of $M$ in $S^{4 m+3}$ is invariant under parallel translation with respect to the normal connection.

Proof. Since $X_{i} \neq 0(i=1, \ldots, q), U_{\nu}=V_{\nu}=W_{\nu}=0$ and $H_{\nu}=0(\nu=$ $3 q+1, \ldots, p)$, we can see that (2.49) and (4.3) imply that the first normal space is spanned by $N_{\alpha}(\alpha=1, \ldots, 3 q)$. For any vector field $X$ tangent to $M$, by means of Lemma 4.4 we have

$$
\nabla_{X}^{\perp} N_{\alpha}=\sum_{A=1}^{p} s_{\alpha A}(X) N_{A}=\sum_{\beta=1}^{3 q} s_{\alpha \beta}(X) N_{\beta},
$$

which show that the first normal space is invariant under parallel translation with respect to the normal connection.

Combining Lemma 4.4 with the results due to Allendoerfer [1] and Erbacher [4] yields that there exists a totally geodesic submanifold $M^{\prime}$ of $S^{4 m+3}$ of dimension $(n+3+3 q)$ such that $M \subset M^{\prime}$. By means of (4.2) and (4.3) with $U_{\nu}=V_{\nu}=W_{\nu}=0(\nu=3 q+1, \ldots, p)$, we can easily see that $M^{\prime}$ is an invariant submanifold of $S^{4 m+3}$ and consequently a $\left(4 m^{\prime}+3\right)$-dimensional sphere for an integer $m^{\prime}$.

Summing up, we may conclude:

Theorem 4.6. Let $M$ be an $(n+3)$-dimensional submanifold isometrically immersed in a unit $(4 m+3)$-sphere $S^{4 m+3}$ to which the structure vector fields $\xi, \eta, \zeta$ are always tangent. Suppose that the normal connection of $M$ in $S^{4 m+3}$ is flat and that the mean curvature vector field is parallel with respect to the normal connection. If $\operatorname{dim}\left(T_{x} M \cap \phi T_{x} M^{\perp} \cap \psi T_{x} M^{\perp} \cap \theta T_{x} M^{\perp}\right)=q(3 q<p)$ at some point $x \in M$, then either $M$ is a totally geodesic, invariant submanifold of $S^{4 m+3}$, or there exists a totally geodesic, invariant submanifold $S^{n+3+3 q}$ of $S^{4 m+3}$ such that $M \subset S^{n+3+3 q}$.

\section{Submanifolds with $L$-flat normal connection}

In this section we try to apply the results which are obtained in the previous sections to submanifolds of a quaternionic projective space.

Let $Q P^{m}$ be a real $4 m$-dimensional quaternionic projective space with quaternionic Kählerian structure $\{J, K, L\}$ and let $\widetilde{g}$ be the Fubini-Study metric which satisfies the Hermitian conditions

(5.1) $\widetilde{g}(J \widetilde{X}, J \widetilde{Y})=\widetilde{g}(\widetilde{X}, \widetilde{Y}), \widetilde{g}(K \widetilde{X}, K \widetilde{Y})=\widetilde{g}(\widetilde{X}, \widetilde{Y}), \widetilde{g}(L \widetilde{X}, L \widetilde{Y})=\widetilde{g}(\widetilde{X}, \widetilde{Y})$.

Then we have

$$
\begin{aligned}
& J^{2}=-I, \quad K^{2}=-I, \quad L^{2}=-I \\
& J=K L=-L K, \quad K=L J=-J L, \quad L=J K=-K J
\end{aligned}
$$


and

$$
\begin{array}{lr}
\widetilde{\nabla}_{\widetilde{X}} J= & r(\widetilde{X}) K-q(\widetilde{X}) L, \\
\widetilde{\nabla}_{\widetilde{X}} K=-r(\widetilde{X}) J \quad+p(\widetilde{X}) L, \\
\widetilde{\nabla}_{\widetilde{X}} L=\quad q(\widetilde{X}) J-p(\widetilde{X}) K
\end{array}
$$

for any vector field $\widetilde{X}$ in $Q P^{m}$, where $\widetilde{\nabla}$ denotes the Riemannian connection with respect to $\widetilde{g}$, and $p, q$ and $r$ are certain local 1 -forms (cf. [5]). It is well known (cf. $[6,15])$ that the quaternionic Kählerian structure $\{J, K, L\}$ is induced from the Sasakian 3-structure $\{\phi, \psi, \theta\}$ of a unit $(4 m+3)$-sphere $S^{4 m+3}$ by the Hopf fibration $\tilde{\pi}: S^{4 m+3} \rightarrow Q P^{m}$. Relations between these structures are given by

$$
\begin{aligned}
& \phi=J^{*}, \quad \psi=K^{*}, \quad \theta=L^{*} \\
& g(X, Y)=\widetilde{g}^{*}(X, Y)+f_{\xi}(X) f_{\xi}(Y)+f_{\eta}(X) f_{\eta}(Y)+f_{\zeta}(X) f_{\zeta}(Y),
\end{aligned}
$$

where $*$ denotes the horizontal lift of indicated quantities. We notice that the structure vector fields $\xi, \eta$ and $\zeta$ are the unit vertical vector fields for the fibration.

Let $M$ be an $n$-dimensional real submanifold of $Q P^{m}$ and construct a $S^{3}$ bundle $\tilde{\pi}^{-1}(M)$ over $M$ in such a way that the following diagram is commutative :

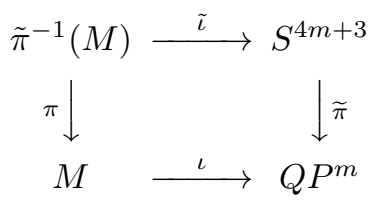

where $\tilde{\iota}: \tilde{\pi}^{-1}(M) \rightarrow S^{4 m+3}$ and $\iota: M \rightarrow Q P^{m}$ are isometric immersions. Then $\tilde{\pi}^{-1}(M)$ is an $(n+3)$-dimensional submanifold of $S^{4 m+3}$ to which the structure vector fields $\xi, \eta$ and $\zeta$ are tangent. Given an orthonormal basis $N_{1}, \ldots, N_{p}$ in $T M^{\perp}$, horizontal lifts $N_{1}^{*}, \ldots, N_{p}^{*}$ are mutually orthonormal normal vector fields to $\tilde{\pi}^{-1}(M)$ with respect to the Riemannian metric $g$ of $\tilde{\pi}^{-1}(M)$ which is induced from that of $S^{4 m+3}$. The transforms for $X \in T M$ and for $N_{A}$ by $\{J, K, L\}$ are, respectively, written by

$$
\begin{aligned}
& J X=\grave{F} X+\sum_{A=1}^{p} \grave{u}^{A}(X) N_{A}, \quad K X=\grave{G} X+\sum_{A=1}^{p} \grave{v}^{A}(X) N_{A}, \\
& L X=\grave{H} X+\sum_{A=1}^{p} \grave{w}^{A}(X) N_{A}, \\
& J N_{A}=-\grave{U}_{A}+\sum_{B=1}^{p} P_{A B}^{J} N_{B}, \quad K N_{A}=-\grave{V}_{A}+\sum_{B=1}^{p} P_{A B}^{K} N_{B}, \\
& L N_{A}=-\grave{W}_{A}+\sum_{B=1}^{p} P_{A B}^{L} N_{B},
\end{aligned}
$$


where $\{\grave{F}, \grave{G}, \grave{H}\}$ and $\left\{P^{J}, P^{K}, P^{L}\right\}$ define endomorphisms of $T M$ and of $T M^{\perp}$, respectively, and $\left\{\grave{U}_{A}, \grave{V}_{A}, \grave{W}_{A}\right\}$ and $\left\{\grave{u}^{A}, \grave{v}^{A}, \grave{w}^{A}\right\}$ are local tangent vector fields and local 1-forms on $M$. Denoting by $\grave{g}$ the Riemannian metric induced on $M$ from that of $Q P^{m}$, we have

$$
\begin{aligned}
& \grave{g}(\grave{F} X, Y)=-\grave{g}(X, \grave{F} Y), \quad \grave{g}(\grave{G} X, Y)=-\grave{g}(X, \grave{G} Y), \\
& \grave{g}(\grave{H} X, Y)=-\grave{g}(X, \grave{H} Y), \\
& P_{A B}^{J}=-P_{B A}^{J}, \quad P_{A B}^{K}=-P_{B A}^{K}, \quad P_{A B}^{L}=-P_{B A}^{L}, \\
& \grave{u}^{A}(X)=\grave{g}\left(\grave{U}_{A}, X\right), \quad \grave{v}^{A}(X)=\grave{g}\left(\grave{V}_{A}, X\right), \quad \grave{w}^{A}(X)=\grave{g}\left(\grave{W}_{A}, X\right)
\end{aligned}
$$

for vector fields $X, Y$ tangent to $M$. Applying $J, K$ and $L$ to (5.5) and making use of (5.2), we can easily obtain the following relations (5.10) and (5.11):

$$
\begin{aligned}
\text { (5.10) } \grave{F}^{2} X & =-X+\sum_{A=1}^{p} \grave{u}^{A}(X) \grave{U}_{A}, \quad \grave{G}^{2} X=-X+\sum_{A=1}^{p} \grave{v}^{A}(X) \grave{V}_{A}, \\
\grave{H}^{2} X & =-X+\sum_{A=1}^{p} \grave{w}^{A}(X) \grave{W}_{A}, \\
\text { (5.11) } \grave{G} \grave{H} X & =\grave{F} X+\sum_{A=1}^{p} \grave{w}^{A}(X) \grave{V}_{A}, \quad \grave{H} \grave{G} X=-\grave{F} X+\sum_{A=1}^{p} \grave{v}^{A}(X) \grave{W}_{A}, \\
\grave{H} \grave{F} X & =\grave{G} X++\sum_{A=1}^{p} \grave{u}^{A}(X) \grave{W}_{A}, \quad \grave{F} \grave{H} X=-\grave{G} X+\sum_{A=1}^{p} \grave{w}^{A}(X) \grave{U}_{A}, \\
\grave{F} \grave{G} X & =\grave{H} X+\sum_{A=1}^{p} \grave{v}^{A}(X) \grave{U}_{A}, \quad \grave{G} \grave{F} X=-\grave{H} X+\sum_{A=1}^{p} \grave{u}^{A}(X) \grave{V}_{A} .
\end{aligned}
$$

Next, applying $J, K$ and $L$ to (5.6) and taking account of (5.2), we have the following relations (5.12)-(5.15):

$$
\begin{gathered}
\text { (5.12) } \grave{F} \grave{U}_{A}=-\sum_{B=1}^{p} P_{A B}^{J} \grave{U}_{B}, \grave{G} \grave{V}_{A}=-\sum_{B=1}^{p} P_{A B}^{K} \grave{V}_{B}, \grave{H} \grave{W}_{A}=-\sum_{B=1}^{p} P_{A B}^{L} \grave{W}_{B}, \\
\text { (5.13) } \grave{G}_{A}=-\grave{W}_{A}-\sum_{B=1}^{p} P_{A B}^{J} \grave{V}_{B}, \quad \grave{H} \grave{U}_{A}=\grave{V}_{A}-\sum_{B=1}^{p} P_{A B}^{J} \grave{W}_{B}, \\
\grave{H} \grave{V}_{A}=-\grave{U}_{A}-\sum_{B=1}^{p} P_{A B}^{K} \grave{W}_{B}, \quad \grave{F} \grave{V}_{A}=\grave{W}_{A}-\sum_{B=1}^{p} P_{A B}^{K} \grave{U}_{B}, \\
\grave{F} \grave{W}_{A}=-\grave{V}_{A}-\sum_{B=1}^{p} P_{A B}^{L} \grave{U}_{B}, \quad \grave{G} \grave{W}_{A}=\grave{U}_{A}-\sum_{B=1}^{p} P_{A B}^{L} \grave{V}_{B}, \\
\text { (5.14) } \grave{g}\left(\grave{U}_{A}, \grave{U}_{B}\right)=\delta_{A B}+\sum_{C=1}^{p} P_{A C}^{J} P_{C B}^{J}, \grave{g}\left(\grave{V}_{A}, \grave{V}_{B}\right)=\delta_{A B}+\sum_{C=1}^{p} P_{A C}^{K} P_{C B}^{K},
\end{gathered}
$$




$$
\grave{g}\left(\grave{W}_{A}, \grave{W}_{B}\right)=\delta_{A B}+\sum_{C=1}^{p} P_{A C}^{L} P_{C B}^{L},
$$

(5.15) $\grave{g}\left(\grave{U}_{A}, \grave{V}_{B}\right)=P_{A B}^{L}+\sum_{C=1}^{p} P_{A C}^{J} P_{C B}^{K}, \grave{g}\left(\grave{V}_{A}, \grave{W}_{B}\right)=P_{A B}^{J}+\sum_{C=1}^{p} P_{A C}^{K} P_{C B}^{L}$,

$$
\grave{g}\left(\grave{W}_{A}, \grave{U}_{B}\right)=P_{A B}^{K}+\sum_{C=1}^{p} P_{A C}^{L} P_{C B}^{J} .
$$

Let $\grave{\nabla}$ and $\grave{\nabla}^{\perp}$ denote the Riemannian connection induced in $M$ and the normal connection of $M$ in $Q P^{m}$, respectively. Denoting by $\grave{H}_{A}$ and $\grave{s}_{A B}$ the Weingarten maps with respect to $N_{A}$ and the connection forms of $\dot{\nabla}^{\perp}$, respectively, we have Gauss and Weingarten formulas for $\widetilde{\nabla}, \grave{\nabla}$ and $\grave{\nabla}^{\perp}$ which are similar to (2.7). Differentiating (5.5) covariantly and using (5.3), we can easily obtain

$$
\begin{aligned}
& \left(\grave{\nabla}_{Y} \grave{F}\right) X=r(Y) \grave{G} X-q(Y) \grave{H} X-\sum_{A=1}^{p} \grave{g}\left(\grave{H}_{A} X, Y\right) \grave{U}_{A}+\sum_{A=1}^{p} \grave{u}^{A}(X) \grave{H}_{A} Y, \\
& \left(\grave{\nabla}_{Y} \grave{G}\right) X=-r(Y) \grave{F} X+p(Y) \grave{H} X-\sum_{A=1}^{p} \grave{g}\left(\grave{H}_{A} X, Y\right) \grave{V}_{A}+\sum_{A=1}^{p} \grave{v}^{A}(X) \grave{H}_{A} Y, \\
& \left(\grave{\nabla}_{Y} \grave{H}\right) X=q(Y) \grave{F} X-p(Y) \grave{G} X-\sum_{A=1}^{p} \grave{g}\left(\grave{H}_{A} X, Y\right) \grave{W}_{A}+\sum_{A=1}^{p} \grave{w}^{A}(X) \grave{H}_{A} Y .
\end{aligned}
$$

Differentiating (5.6) covariantly and using (5.3), we have the following relations (5.17) and (5.18):

$$
\begin{aligned}
& \grave{\nabla}_{X} \grave{U}_{A}=r(X) \grave{V}_{A}-q(X) \grave{W}_{A}+\grave{F} \grave{H}_{A} X-\sum_{B=1}^{p} P_{A B}^{J} \grave{H}_{B} X+\sum_{B=1}^{p} \grave{s}_{A B}(X) \grave{U}_{B}, \\
& \grave{\nabla}_{X} \grave{V}_{A}=-r(X) \grave{U}_{A}+p(X) \grave{W}_{A}+\grave{G} \grave{H}_{A} X-\sum_{B=1}^{p} P_{A B}^{K} \grave{H}_{B} X+\sum_{B=1}^{p} \grave{s}_{A B}(X) \grave{V}_{B}, \\
& \grave{\nabla}_{X} \grave{W}_{A}=q(X) \grave{U}_{A}-p(X) \grave{V}_{A}+\grave{H} \grave{H}_{A} X-\sum_{B=1}^{p} P_{A B}^{L} \grave{H}_{B} X+\sum_{B=1}^{p} \grave{s}_{A B}(X) \grave{W}_{B},
\end{aligned}
$$

$$
\begin{aligned}
\grave{\nabla} \grave{X}_{X} P_{A B}^{J} & :=\nabla_{X} P_{A B}^{J}+\sum_{C=1}^{p} P_{C B}^{J} \grave{s}_{C A}(X)+\sum_{C=1}^{p} P_{A C}^{J} \grave{s}_{C B}(X) \\
& =r(X) P_{A B}^{K}-q(X) P_{A B}^{L}+\grave{g}\left(\grave{U}_{A}, \grave{H}_{B} X\right)-\grave{u}^{B}\left(\grave{H}_{A} X\right), \\
\grave{\nabla} \frac{1}{X} P_{A B}^{K} & :=\nabla_{X} P_{A B}^{K}+\sum_{C=1}^{p} P_{C B}^{K} \grave{s}_{C A}(X)+\sum_{C=1}^{p} P_{A C}^{K} \grave{s}_{C B}(X)
\end{aligned}
$$




$$
\begin{aligned}
& =-r(X) P_{A B}^{J}+p(X) P_{A B}^{L}+\grave{g}\left(\grave{V}_{A}, \grave{H}_{B} X\right)-\grave{v}^{B}\left(\grave{H}_{A} X\right), \\
\grave{\nabla}_{X}^{\perp} P_{A B}^{L}: & =\nabla_{X} P_{A B}^{L}+\sum_{C=1}^{p} P_{C B}^{L} \grave{s}_{C A}(X)+\sum_{C=1}^{p} P_{A C}^{L} \grave{S}_{C B}(X) \\
& =q(X) P_{A B}^{J}-p(X) P_{A B}^{K}+\grave{g}\left(\grave{W}_{A}, \grave{H}_{B} X\right)-\grave{w}^{B}\left(\grave{H}_{A} X\right) .
\end{aligned}
$$

On the other hand, $Q P^{m}$ is of constant $Q$-sectional curvature 4 and so the curvature tensor $\widetilde{R}$ of $Q P^{m}$ has the following form (cf. [5]):

$$
\begin{aligned}
\widetilde{R}(\widetilde{X}, \widetilde{Y}) \widetilde{Z}= & \tilde{g}(\widetilde{Y}, \widetilde{Z}) \widetilde{X}-\tilde{g}(\widetilde{X}, \widetilde{Z}) \widetilde{Y} \\
& +\tilde{g}(J \widetilde{Y}, \widetilde{Z}) J \widetilde{X}-\tilde{g}(J \widetilde{X}, \widetilde{Z}) J \widetilde{Y}-2 \tilde{g}(J \widetilde{X}, \widetilde{Y}) J \widetilde{Z} \\
& +\tilde{g}(K \widetilde{Y}, \widetilde{Z}) K \widetilde{X}-\tilde{g}(K \widetilde{X}, \widetilde{Z}) K \widetilde{Y}-2 \tilde{g}(K \widetilde{X}, \widetilde{Y}) K \widetilde{Z} \\
& +\tilde{g}(L \widetilde{Y}, \widetilde{Z}) L \widetilde{X}-\tilde{g}(L \widetilde{X}, \widetilde{Z}) L \widetilde{Y}-2 \tilde{g}(L \widetilde{X}, \widetilde{Y}) L \widetilde{Z}
\end{aligned}
$$

Thus, using (5.5) and (5.6), we have the following Codazzi and Ricci equations (5.19) and (5.20), respectively:

$$
\begin{aligned}
& \left(\grave{\nabla}_{X} \grave{H}_{A}\right) Y-\left(\grave{\nabla}_{Y} \grave{H}_{A}\right) X \\
= & \sum_{B=1}^{p}\left\{\grave{s}_{A B}(X) \grave{H}_{B} Y-\grave{s}_{A B}(Y) \grave{H}_{B} X\right\} \\
& -\grave{g}\left(\grave{U}_{A}, Y\right) \grave{F} X+\grave{g}\left(\grave{U}_{A}, X\right) \grave{F} Y-2 \grave{g}(\grave{F} X, Y) \grave{U}_{A} \\
& -\grave{g}\left(\grave{V}_{A}, Y\right) \grave{G} X+\grave{g}\left(\grave{V}_{A}, X\right) \grave{G} Y-2 \grave{g}(\grave{G} X, Y) \grave{V}_{A} \\
& -\grave{g}\left(\grave{W}_{A}, Y\right) \grave{H} X+\grave{g}\left(\grave{W}_{A}, X\right) \grave{H} Y-2 \grave{g}(\grave{H} X, Y) \grave{W}_{A},
\end{aligned}
$$

(5.20) $\grave{R}^{\perp}(X, Y) N_{A}$

$$
\begin{aligned}
= & \sum_{B=1}^{p}\left\{\grave{g}\left(\left(\grave{H}_{A} \grave{H}_{B}-\grave{H}_{B} \grave{H}_{A}\right) X, Y\right)\right. \\
& +\grave{g}\left(\grave{U}_{A}, Y\right) \grave{g}\left(\grave{U}_{B}, X\right)-\grave{g}\left(\grave{U}_{A}, X\right) \grave{g}\left(\grave{U}_{B}, Y\right)-2 \grave{g}(\grave{F} X, Y) P_{A B}^{J} \\
& +\grave{g}\left(\grave{V}_{A}, Y\right) \grave{g}\left(\grave{V}_{B}, X\right)-\grave{g}\left(\grave{V}_{A}, X\right) \grave{g}\left(\grave{V}_{B}, Y\right)-2 \grave{g}(\grave{G} X, Y) P_{A B}^{K} \\
& \left.+\grave{g}\left(\grave{W}_{A}, Y\right) \grave{g}\left(\grave{W}_{B}, X\right)-\grave{g}\left(\grave{W}_{A}, X\right) \grave{g}\left(\grave{W}_{B}, Y\right)-2 \grave{g}(\grave{H} X, Y) P_{A B}^{L}\right\} N_{B},
\end{aligned}
$$

where $\grave{R}^{\perp}$ denotes the curvature tensor of the normal connection $\grave{\nabla}^{\perp}$. Here we notice that if $M$ is an invariant submanifold of $Q P^{m}$, then $M$ is totally geodesic (cf. [6]) and $\grave{U}_{A}=\grave{V}_{A}=\grave{W}_{A}=0(A=1, \ldots, p)$.

If $R^{\perp}$ satisfies

$$
\begin{aligned}
& \grave{R}^{\perp}(X, Y) N_{A} \\
= & \sum_{B=1}^{p}\left\{-2 \grave{g}(\grave{F} X, Y) P_{A B}^{J}-2 \grave{g}(\grave{G} X, Y) P_{A B}^{K}-2 \grave{g}(\grave{H} X, Y) P_{A B}^{L}\right\} N_{B}
\end{aligned}
$$


and

$$
\begin{aligned}
& \grave{\nabla}_{X}^{\perp} P_{A B}^{J}=r(X) P_{A B}^{K}-q(X) P_{A B}^{L}, \\
& \grave{\nabla}_{X}^{\perp} P_{A B}^{K}=-r(X) P_{A B}^{J}+p(X) P_{A B}^{L}, \\
& \grave{\nabla}_{X}^{\perp} P_{A B}^{L}=q(X) P_{A B}^{J}-p(X) P_{A B}^{K},
\end{aligned}
$$

then the normal connection of $M$ is said to be lift-flat or briefly $L$-flat. It is well known ([17, Theorem 3.5, p. 431]) that the normal connection of $M$ is $L$-flat if and only if the normal connection of $\widetilde{\pi}^{-1}(M)$ is flat. In [17], when (5.22) is satisfied, the structure induced in the normal bundle of $M$ in $Q P^{m}$ is said to be parallel.

Let $H_{A}, \mu$ and $\grave{\mu}$ be the Weingarten map with respect to $N_{A}^{*}$, the mean curvature vector field of $\tilde{\pi}^{-1}(M)$ and of $M$, respectively. Then the following relations are known (cf. [16]):

$$
\begin{gathered}
H_{A} X^{*}=\left(\grave{H}_{A} X\right)^{*}+\grave{g}\left(\grave{U}_{A}, X\right)^{*} \xi+\grave{g}\left(\grave{V}_{A}, X\right)^{*} \eta+\grave{g}\left(\grave{W}_{A}, X\right)^{*} \zeta, \\
\operatorname{tr} H_{A}=\left(\operatorname{tr} \grave{H}_{A}\right)^{*},(A=1, \ldots, p) \\
\nabla_{X^{*}}^{\perp} \mu=\frac{n}{n+3}\left(\grave{\nabla}_{X}^{\perp} \grave{\mu}\right)^{*}, \\
P_{A B}^{J}{ }^{*}=s_{A B}(\xi), \quad P_{A B}^{K}{ }^{*}=s_{A B}(\eta), \quad P_{A B}^{L}{ }^{*}=s_{A B}(\zeta) .
\end{gathered}
$$

It is clear from (5.23) that $M$ is minimal if and only if $\tilde{\pi}^{-1}(M)$ is minimal (cf. [16]). Finally we verify

Theorem 5.1. Let $M$ be an $n$-dimensional real minimal submanifold of $Q P^{m}$. If the normal connection of $M$ in $Q P^{m}$ is $L$-flat and at some point of $x \in M$, $\operatorname{dim}\left(T_{x} M \cap J T_{x} M^{\perp} \cap K T_{x} M^{\perp} \cap L T_{x} M^{\perp}\right)=q(3 q<p:=4 m-n)$, then either $M$ is a totally geodesic, invariant submanifold of $Q P^{m}$ or there exist a real $(n+3 q)$-dimensional totally geodesic, invariant submanifold $Q P^{(n+3 q) / 4}$ of $Q P^{m}$ such that $M \subset Q P^{(n+3 q) / 4}$.

Proof. Since $\operatorname{dim}\left(T_{x} M \cap J T_{x} M^{\perp} \cap K T_{x} M^{\perp} \cap L T_{x} M^{\perp}\right)=q$ and the Riemannian metric $\widetilde{g}$ satisfies the Hermitian conditions, there exist mutually orthonormal normal vectors $n_{1}, \ldots, n_{3 q}$ such that

$$
J_{x} n_{1}=K_{x} n_{q+1}=L_{x} n_{2 q+1}, \ldots, J_{x} n_{q}=K_{x} n_{2 q}=L_{x} n_{3 q}
$$

constitute an orthonormal basis for $T_{x} M \cap J T_{x} M^{\perp} \cap K T_{x} M^{\perp} \cap L T_{x} M^{\perp}$. We extend $n_{1}, \ldots, n_{3 q}$ to local fields $N_{1}, \ldots, N_{3 q}$ in $T M^{\perp}$ and choose $N_{3 q+1}, \ldots, N_{p}$ in $T M^{\perp}$ so that $N_{1}, \ldots, N_{3 q}, N_{3 q+1}, \ldots, N_{p}$ are mutually orthonormal. Then $N_{1}^{*}, \ldots, N_{3 q}^{*}, N_{3 q+1}^{*}, \ldots, N_{p}^{*}$ are orthonormal vector fields in $T \widetilde{\pi}^{-1}(M)^{\perp}$. Let $y \in \tilde{\pi}^{-1}(x)$, then

$$
\operatorname{dim}\left(T_{y} \widetilde{\pi}^{-1}(M) \cap \phi_{y} T_{y} \tilde{\pi}^{-1}(M)^{\perp} \cap \psi_{y} T_{y} \tilde{\pi}^{-1}(M)^{\perp} \cap \theta_{y} T_{y} \tilde{\pi}^{-1}(M)^{\perp}\right)=q
$$

because of (5.4). Furthermore, $\tilde{\pi}^{-1}(M)$ is minimal in $S^{4 m+3}$ because of (5.24) and the normal connection of $\tilde{\pi}^{-1}(M)$ is flat. Thus, by means of Theorem $4.6, \tilde{\pi}^{-1}(M)$ is a totally geodesic invariant submanifold $S^{n+3}$ of $S^{4 m+3}$, 
or there exists a totally geodesic invariant submanifold $S^{n+3+3 q}$ such that $\tilde{\pi}^{-1}(M) \subset S^{n+3+3 q}$. $S^{n+3+3 q}$ is a $S^{3}$-bundle over a quaternionic projective space $Q P^{(n+3 q) / 4}$ of a real $(n+3 q)$-dimension and $\{\xi, \eta, \zeta\}$ are the unit vertical vector fields of the $S^{3}$-bundle. Thus the immersion : $Q P^{(n+3 q) / 4} \rightarrow Q P^{m}$ is compatible with the Hopf fibration $\widetilde{\pi}: S^{4 m+3} \rightarrow Q P^{m}$. Since $S^{n+3+3 q}$ is a totally geodesic submanifold in $S^{4 m+3},(5.23)$ implies that $Q P^{(n+3 q) / 4}$ is a totally geodesic, invariant submanifold of $Q P^{m}$. This completes the proof.

\section{References}

[1] C. B. Allendoerfer, Rigidity for spaces of class greater than one, Amer. J. Math. Soc. 61 (1939), 633-644.

[2] T. E. Cecil, Geometric applications of critical point theory to submanifolds of complex projective space, Nagoya Math J. 55 (1974), 5-31.

[3] B. Y. Chen, Geometry of Submanifolds, Marcel Dekker Inc., New York, 1973.

[4] J. Erbacher, Reduction of the codimension of an isometric immersion, J. Differential Geometry 5 (1971), 333-340.

[5] S. Ishihara, Quaternion Kaehlerian manifolds, J. Differential Geometry 9 (1974), 483500 .

[6] S. Ishihara and M. Konish, Differential Geometry of Fibred Spaces, Study Group of Differential Geometry, Tokyo, 1973.

[7] T. Kashiwada, A note on a Riemannian space with Sasakian 3-structure, Natur. Sci. Rep. Ochanomizu Univ. 22 (1971), 1-2.

[8] S. Kawamoto, Codimension reduction for real submanifolds of a complex hyperbolic space, Rev. Mat. Univ. Complut. Madrid 7 (1994), no. 1, 119-128.

[9] H. S. Kim and J. S. Pak, Codimension reduction for real submanifolds of quaternionic hyperbolic space, Acta Math. Hungar. 121 (2008), no. 1-2, 21-33.

[10] Y. Y. Kuo, On almost contact 3-structure, Tohoku Math. J. 22 (1970), 325-332.

[11] J.-H. Kwon and J. S. Pak Codimension reduction for real submanifolds of quaternionic projective space, J. Korean Math. Soc. 36 (1999), no. 1, 109-123.

[12] H. B. Lawson, Jr., Rigidity theorems in rank-1 symmetric spaces, J. Differential Geometry 4 (1970), 349-357.

[13] M. Okumura, Reducing the codimension of a submanifold of a complex projective space, Geom. Dedicata 13 (1982), no. 3, 277-289.

[14] _ Codimension reduction problem for real submanifold of complex projective space, Differential geometry and its applications (Eger, 1989), 573-585, Colloq. Math. Soc. János Bolyai, 56, North-Holland, Amsterdam, 1992.

[15] J. S. Pak, Real hypersurfaces in quaternionic Kaehlerian manifolds with constant $Q$ sectional curvature, Kodai Math. Sem. Rep. 29 (1977), no. 1-2, 22-61.

[16] Y. Shibuya, Real submanifolds in a quaternionic projective space, Kodai Math. J. 1 (1978), no. 3, 421-439.

[17] S. Tachibana and W. N. Yu, On a Riemannian space admitting more than one Sasakian structures, Tohoku Math. J. 22 (1970), 536-540.

HYANG SOOK KIM

Department of Applied Mathematics

Institute OF BASIC SCIENCE

INJE UNIVERSITY

KimhaE 621-749, KoreA

E-mail address: mathkim@inje.ac.kr 
JIN SUK PAK

KyungPoOK National University

DAEGU 702-701, KorEA

E-mail address: jspak@knu.ac.kr 